\title{
Project Risk Management Implementation in SMEs: A Case Study from Italy
}

\author{
Priscila Ferreira de Araujo Lima ${ }^{1}$ and Chiara Verbano ${ }^{1 *}$
}

\begin{abstract}
Despite its strategical importance, the diffusion of Project Risk Management (PRM) is limited mainly to large companies, leaving a lack of empirical evidence addressing small and medium-sized enterprises (SMEs). Therefore, the objective of this paper is to analyse how to adopt PRM in SMEs with a positive cost-benefit ratio, considering risk management (RM) phases, activities, tools, instruments, procedures and organisational aspects that enable the effective implementation of PRM in SMEs. To identify the fundamental dimensions to insert in the framework, a systematic literature review was performed, and a pilot case study was conducted to test the validity of the empirical framework and improve it. Moreover, after the case analysis, the benefits obtained and the difficulties faced through the adoption of PRM are presented and discussed.
\end{abstract}

Keywords: project risk management; project management; small and medium-sized enterprises; empirical framework; case study

Submitted: August 21 $1^{\text {st }}, 2018$ / Approved: March 22 2019

\section{Introduction}

According to ISO 31000:2009, risk is defined as the effect, in terms of positive or negative deviation from the expected outcome, resulting from uncertainty on targeted objectives. Risks can affect organisations in terms of economic performance, business continuity, reputation, environmental and social outcomes; therefore the management of risks supports organisations in reaching their targeted objectives, reducing potential losses and exploring new opportunities in an environment full of uncertainties (ISO 31000, 2009; Radner \& Shepp, 1996). Consequently, all types of organisations are taking a greater interest in risk management (RM), recognising the deriving benefits.

The aim of RM is to reduce the potential for risks and mitigate the impact of possible losses (Bajo et al., 2012). RM is a continuous process of defining objectives, identifying sources of uncertainties, measuring probability and severity of the potential consequences and formulating managerial responses to risks and opportunities (Henschel, 2006).

The literature classifies RM into 9 different streams, based on the different approaches used, the risks considered, the techniques applied, the methodologies proposed and the fields of application: Clinical RM, Disaster RM, Engineering RM, Enterprise RM, Financial RM, Insurance RM, Project RM, Strategic RM and Supply Chain RM (Verbano \& Venturini, 2011).

Project RM (PRM) is an integral part of Project Management (PM), where PM is the application of methods, knowledge, tools and techniques to a project, integrating the various phases of a project's lifecycle in order to achieve its goal (ISO 21500:2012; PMI, 2017). The PM process is divided into five phases (i.e., initiation, planning, execution, monitoring \& control and closure) (ISO 21500:2012), and its main purpose is to increase the organisational value (Dalcher, 2012). Most companies are aware of the importance of a structured approach to
PM (Miklosik, 2015) and of the increased effectiveness and efficiency of human effort in the organisation that is obtained through PM (Fernando et al., 2018), opting then for its adoption.

When a project is implemented in a company, it is essential to design all of the project's lifecycle and identify all the possible sources of risks that can be technical-operational, organisational, financial and strategic (Badri, 2015). To ensure project success in today's competitive environment, an organisation must manage these project risks effectively, even though various difficulties are experienced (Shenhar et al., 2007; Srivannaboon \& Milosevic, 2006). Through the identification of such risks, the managers are able to study the possible positive and negative outcomes and define the correct response to treat them in order to assure the success of the entire project. Commonly, in most types of organisations, many efforts of managers are expended on dealing with project risk-related issues (Fernando et al., 2018). PRM activities, tools and techniques and organisational aspects offer an approach to manage these risks proactively in order to improve the projects' probability of success.

PRM contributes to identify the project objectives, improve the project monitoring and control, improve communication among the project stakeholders, facilitate decision-making and increase the chances of project success (PMI, 2013; Raz \& Michael, 2001). It is a subset of PM, both in the Project Management Institute (PMI) framework and in the ISO 21500 (ISO 21500:2012; PMI, 2013), composed of four phases: risk identification, risk analysis, risk treatment and risk monitor \& control (PMI, 1996).

The aim of PRM is to systematically identify, analyse, treat and monitor \& control project-related risks by decreasing the probability and impact of occurrence of negative ones (threats) as well as increasing the probability and impact of occurrence of positive events (opportunities) during the project (Borge, 2002; PMI, 2013).

(1) Department of Management and Engineering, University of Padova, Stradella San Nicola 3, 36100, Vicenza, Italy.

*Corresponding author: chiara.verbano@unipd.it 
Given its importance, in the last years, many companies, from different types of industries, have adopted PRM. Using the appropriate tools, PRM provides an effective means for managing a complex project against time, cost and quality non-achievement (Dey, 2012).

Also, Elkington and Smallman (2000) claim that PRM is essential for the project's success. They have identified that the most successful projects implemented more RM practices. In fact, the earlier RM is initiated, the more successful a project becomes.

RM process activities and the criteria for choosing among risk techniques have been extensively studied and implemented in both literature and practice. However, these criteria usually do not consider either the characteristics of the project and of its surrounding environment or the attitude of an organisation towards risk (Cagliano, Grimaldi, \& Rafele, 2015).

Despite its strategical importance, PRM diffusion is limited mainly to large companies, leaving a lack of empirical evidence addressing small and medium-sized enterprises (SMEs) (Kim \& Vonortas, 2014). The SMEs vulnerability, because of a combination of resource-related constraints (i.e., human, technical and financial constraints), low bargaining power and sensitiveness to business risk and competition, leads to an additional need of PRM adoption (Blanc-Alquier \& Lagasse-Tignol, 2006; Dallago, Guglielmetti, \& Rondinelli, 2012).

However, different frameworks of RM are necessary, depending on the size of the project to facilitate the RM application in SMEs (Turner, Ledwith, \& Kelly, 2010). According to Marcelino-Sádaba et al. (2014), SMEs need less bureaucratic PM models, with perhaps a different toolset to traditional versions designed for medium or large projects, which could facilitate the RM throughout the project lifecycle.

With the aim to investigate the diffusion of PRM among small companies, a bibliographic search was conducted in the two most widespread academic databases, Scopus and Web of Science, using different combinations of the following keywords: 'risk management', 'SMEs' and 'small business'. After a careful selection process, in which the proceeding papers, editorial materials, non-English language papers, non-pertinent articles were excluded, only six papers about PRM in SMEs were found. All six papers were empirical studies (i.e., three case studies and three model proposal and testing). Three of the studies were applied in the industrial sector, two were applied in the service sector and one was applied in both sectors. Only half of the papers contemplated all project risk types and, as for the PRM phases, only in two of them all phases were applied. Despite its overmentioned importance, PRM is neither much studied nor widespread in SMEs (Marcelino-Sádaba et al., 2014), and, given the abovementioned results, it was possible to conclude that there is a significant gap to be fulfilled with new researches.

\section{Objectives and Methodology}

The objective of this paper is to analyse how to adopt PRM in SMEs with a positive cost-benefit ratio, considering RM phases, activities, tools, instruments, procedures and other organisational aspects that enable the effective implementation of PRM in SMEs. Given the nature of the objective of this research, an exploratory and explanatory research through the case study methodology has been chosen, as it is the most appropriate one for this type of study (Yin, 1994). To this extent, an empirical framework of analysis has been specifically developed to conduct a multiple case study focused on projects in SMEs, in which PRM was adopted with a positive cost-benefit ratio. In this paper, a pilot case study will be presented, aiming specifically at testing the validity of the empirical framework and improving it; moreover, first indications to promote PRM adoption in SMEs will be grasped from the analysis of the pilot case.

To build the research protocol, firstly, well-known books and significant articles about research methodologies were consulted. After that, consolidated manuals about PM and PRM, as well as empirical papers about the adoption of PRM by different types of companies, were analysed in order to collect the fundamental dimensions to be inserted in the theoretical framework. All the collected information was adapted to the SMEs context and summarised to support the questionnaire creation. The steps of the methodology and their specific aims are presented in Figure 1.

Figure 1: Construction of the research protocol

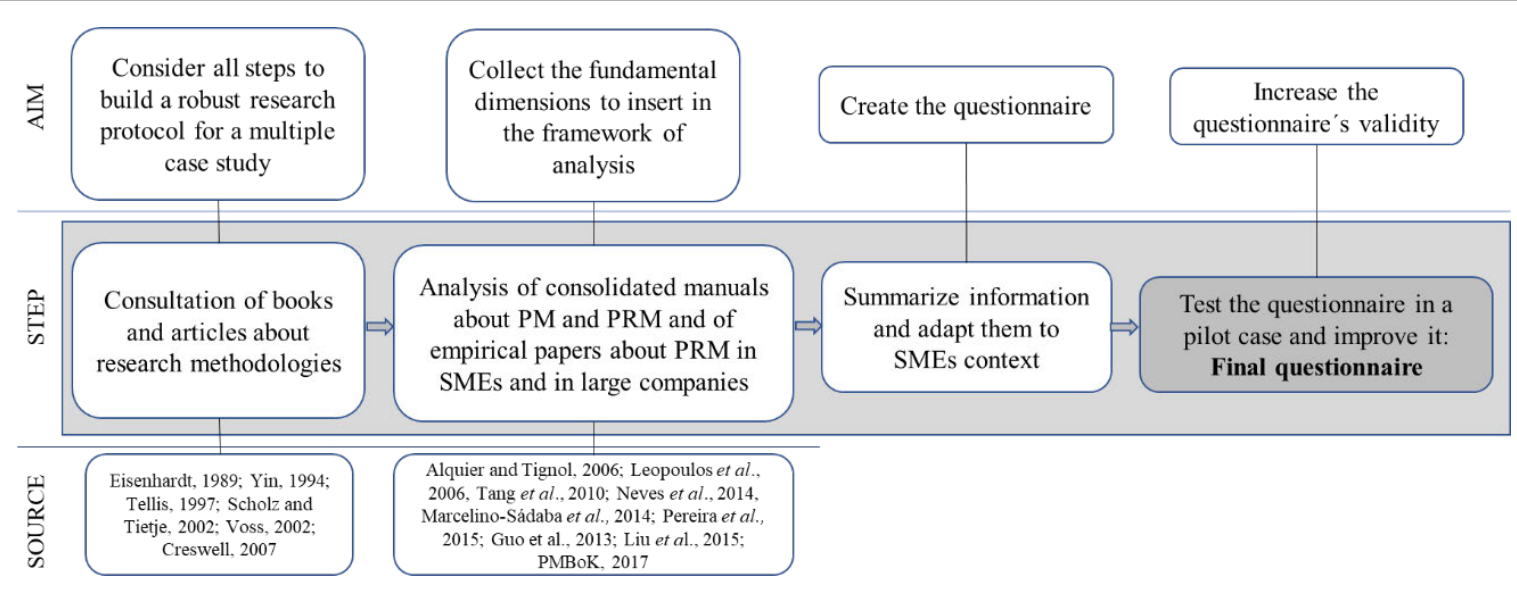


The resulting questionnaire is divided into eight sections: (1) Company profile and respondents; (2) Project overview (i.e., the main characteristics, objectives and specific items useful to understand the innovativeness and the complexity of the project); (3) PRM process and organisation (i.e., the phases and roles involved); (4) PRM identification phase; (5) PRM analysis phase; (6) PRM treatment phase; (7) PRM monitor \& control phase. (8) Outcomes of the PRM adoption (i.e., the benefits, time and costs of implementation, as well as the enabling factors and obstacles). In each of these sections, the activities performed, the tools and techniques used, the results obtained and the main difficulties encountered were also questioned.

The pilot project was selected based on the adoption of PRM by a SME, the type of industry in which it was implemented (i.e., ICT services) and the availability and knowledge of the project manager and other members of the project team. Furthermore, to ensure the project's internal validity through triangulation and to increase data reliability (Voss, Tsikriktsis, \& Frohlich, 2002), the results of the indepth interviews with multiple respondents were integrated with the analysis of documents related to the PRM plan and outcomes, which were delivered to the researchers.

\section{Results}

\section{Company profile and project overview}

For this pilot case study, a small-sized consultancy company - organised by projects - was selected. The company employs 48 people and has a turnover of five million Euros. Moreover, its main activity is the development of Customer Relationship Management (CRM) systems that focus on user adoption (i.e., the spontaneous and profitable utilisation of CRM by its users). To protect the company's confidentiality, moving forward, the company will be referred to as 'Alpha'.

The analysed project consisted of implementing a new CRM system to an external client that belonged to the automotive industry and had the ambitious objective of increasing its annual income by $30 \%$. The project cost 60,000 Euros and involved seven people for seven months. Two members of the project team were interviewed: firstly, by the project manager and, secondly, by the manager's assistant, who played an important role in verifying and integrating the previously collected data.

In order to understand the main characteristics of the project, the interviewees were asked to classify the project's innovativeness and complexity on a Likert-type scale, from 1 (very low) to 5 (very high). The technological innovativeness was considered to be very high, while the client's market innovativeness was considered to be low. The complexity of the PM was rated as 'medium', while the project's relevance for the company's strategy was considered very high since both the project manager and the assistant believed that having success in this project may allow the organisation to enter into a new market. The average score obtained from the project's innovativeness and complexity is 3.75 (as displayed in Figure 2), highlighting a potential medium-high level of risk that is usually associated with the abovementioned characteristics.
Figure 2: Project's innovativeness and complexity evaluation

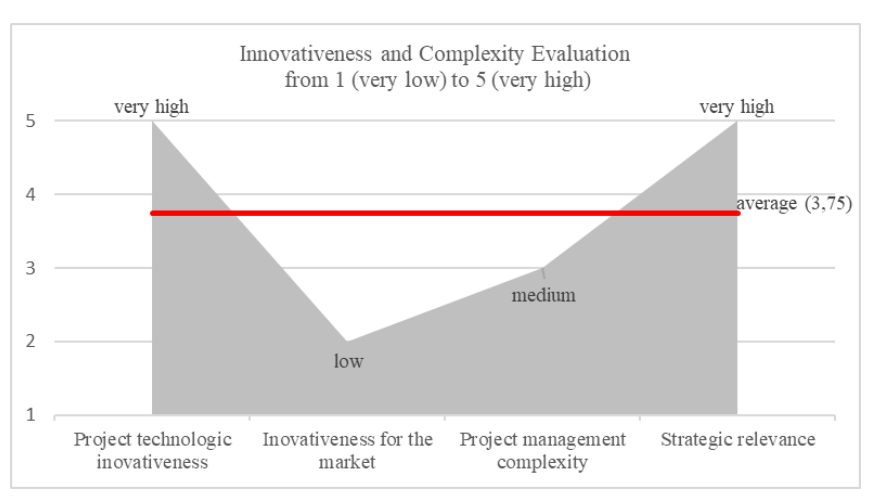

PRM process and organisation

The project manager was responsible for the PRM. The assistant helped the project manager throughout the process, and the sales manager was involved during the identification, the analysis and the monitor \& control phases. Table 1 displays the general information about the PRM process and the organisation adopted in the project.

Table 1: PRM overview in the analysed case

\begin{tabular}{l|l}
\hline \multicolumn{2}{l}{ PRM overview } \\
\hline Implemented PRM phases & $\begin{array}{l}\text { identification, analysis, treatment, mo- } \\
\text { nitor \& control }\end{array}$ \\
\hline PM phases with PRM adoption & initiation, planning, execution, closure \\
\hline Responsible for PRM & $\begin{array}{l}\text { project manager (supported by his as- } \\
\text { sistant) }\end{array}$ \\
\hline PRM team & $\begin{array}{l}\text { project manager (PRM responsible) } \\
\text { project manager assistant } \\
\text { sales manager }\end{array}$ \\
\hline Risk owners identified & yes \\
\hline
\end{tabular}

\section{Risk identification and analysis}

In this project, as in all other projects of Alpha, a preliminary analysis was performed. The analysis consisted of a three-day evaluation, with the aim of defining the actual picture of the company's infrastructure and understanding the possible interactions between the client's information system and the CRM system to be implemented. Thus, the outcomes of this analysis served as an input for the project planning.

During this preliminary analysis, the activities to be performed were ordered by importance in a matrix (deriving from a cost-benefit analysis), and the possible risks that may be faced during the project were identified and analysed. Therefore, the first two phases of the PRM process, risk identification and risk analysis, were proactively performed in this first PM phase (i.e., initiation).

In particular, for the risk identification, the activities performed - mainly to check the feasibility - were context analysis, stakeholder analysis and risks and opportunities identification, while the main tools used were brainstorming, interviews with experts, SWOT Analysis and the 5 Whys technique. No relevant, technical-operational 
risks were identified; however, if there were any identified in this preliminary analysis, the company would not have accepted the project. In addition to that, as a mitigating action, a clause in the contract signed by the client allows Alpha to interrupt the project if any risks related to the IT structure emerges.

Strategic risks, however, were identified (e.g., the possible issues impeding an effective user adoption), in addition to few organisational risks (i.e., the operational risks); for example, there was a risk of assigning part of the project to an inexperienced or low-skilled software engineer, which, in turn, opens the risk of not delivering the project in time. The client was considered to be reliable from a financial point of view; thus, no relevant financial risks were considered.

Besides the risks, an opportunity was also identified: to turn this project into a model for future clients in the automotive industry. For this reason, the project manager devoted all his effort towards obtaining a specific CRM model for this industry by the end of the project.

The main obstacle encountered in this phase was understanding what the main risks for the client were. The project manager and the assistant, who were responsible for the PRM, were very concerned about the risk of not satisfying the client because of the potential difficulties that could be faced during the CRM user adoption. Such difficulties could not only jeopardise the project but also threaten the opportunities of making the project a successful case and attracting new clients in the market.

During the risk analysis, the project team focused on analysing the consequences of the risks identified as well as the risks that could arise during the execution of the project. The tools and techniques adopted in this phase were the 5 Whys technique and a cost-benefit analysis, obtaining a qualitative evaluation of the identified risks. No risk prioritisation was done in the project. The risk related to the user adoption and the eventual lack of skills of a team member were analysed. For example, the user adoption was considered a risk with a significant negative impact for the company's strategy in case it occurred, while the lack of knowledge of one of the software engineers could incur into extra working days, which means a loss of money.

Despite all the analysis conducted, they did not create a risk register and, because of a CEO's decision, the risks were not reported in any document. Several difficulties were faced during the risk analysis, and the main ones were the possible unreliable information given by the client and the need to deal with unexpected risk. Given the activities and connected risks, the project manager approved the preliminary analysis and officially started the project. During the project lifecycle, the project manager had the responsibility of having these initially identified risks under control.

\section{Risk treatment}

The definition of risk acceptance threshold was based on the consultants' feelings and knowledge, without any type of objective or quantitative analysis threshold.

Firstly, risk treatment was performed in the preliminary analysis. With the aim to assure project success, every source of technical impediments related to the client infrastructure was accurately considered and, if detected, the project would have been excluded to avoid the risks. Moreover, to transfer other potential technical risks emerging during the project, a clause in the contract allows Alpha to interrupt the project if any risk related to the IT structure, and not initially individuated, emerges.

Two specific techniques were used to complete the risk treatment during the project implementation: risk mitigation and, more widely, risk transfer. Both the project manager and the assistant recognised that these actions should be planned in advance; nevertheless, as in most of the company's projects, they managed both the risk mitigation and the risk transfer 'live' (i.e., following a reactive approach). For example, in this project, an SMS alert system was implemented in the CRM with a particular template and a timer. While this SMS system was being built, one of the client's managers was replaced by another one, who was not satisfied with the final template nor with the timer configuration and asked for modifications. These changes imply extra working hours for Alpha's engineers; therefore, Alpha transferred the risk of extra costs to the user since the specifications of the user requirements were different from what was specified in the contract. As a result, the sales manager created a change request, and the client payed for it.

Another risk that occurred in the project was related to the lack of skills of one of the software engineers. During the second day of the project, a software engineer - as a member of the project team - asked the project manager to leave the project because he was not capable of developing all of the required activities. Since this was an expected risk, the project manager had already considered another person and made a substitution in the project team; however, this replacement could not be done immediately so, in the end, two working-days were lost (i.e., approximately 1,500 Euros). In this case the risk was partially mitigated but not completely avoided.

A significant difficulty faced in this phase was to involve the client, given his poor availability.

\section{Risk monitoring \& control}

During this phase, the main two activities carried on were change request monitoring and risk trigger monitoring. To this extent, a risk tracking tool, called 'GIRA', was developed by the software engineers of Alpha using an Agile logic. Through 'GIRA', the project team was able to keep track of any change request and the eventual problems that emerged during the project execution. This tool was also useful to support the project manager to discuss with the client and decide which treatment action to take when needed. The main difficulties faced during this phase were the lack of time, the need of dealing with non-calculated risks and the lack of information provided by the client.

Once the project finished, a meeting involving all the project members was held at Alpha, during which they discussed and reported the lessons learned about how to avoid in the future the same difficulties and problems encountered in this project; these results were not documented. 


\section{PRM Outcomes}

According to the consultants, the use of PRM in this project had a very high impact on improving the project planning, increasing the probability of project success and increasing the client's trust. Moreover, the project manager and the assistant also affirmed that PRM has highly contributed in reducing the risk impact and improving the project's performance. Through PRM application, the main risks were avoided, and a significant strategic opportunity was identified; thus, pursing this opportunity gave extra motivation for the project team. Besides that, both interviewees affirmed that PRM supported them in the decision-making process, but it was not very significant because there was no risk register, and risk evaluation was not performed in a systematic way. However, the project manager believes that, if the PRM process were more structured and the risk analysis were deeper, the project team would have more reliable information about the project that could considerably contribute in the decision-making process; therefore, potential PRM outcomes are even higher than the actual outcomes.
The adoption of PRM had a low impact on improving the evaluation of the budget reserve and on the budget controlling, since there was not a quantitative risk evaluation; however, both respondents believed that these outcomes could benefit from an improved application of PRM.

The project manager and the assistant believed the PRM benefits obtained overcame the implementation costs (i.e., approximately 3,000 Euros) and the time spent in its analysis (i.e., 5 working days). They also believed that it should be implemented in all types of projects. They further pointed out that the strategic nature of the project gave a strong motivation for the PRM adoption. Among the main obstacles faced during the PRM implementation were: risks not clearly identified at the beginning of the project, difficult communications with the client and time constraints. Nevertheless, the opportunity identified in the preliminary analysis was successfully achieved; thus, by the time of the project's closure, Alpha developed the main structure of a specific CRM model for the automotive industry. Figure 3 summarises the results obtained from the pilot case, according to the framework of analysis.

Figure 3: PRM process and outcomes

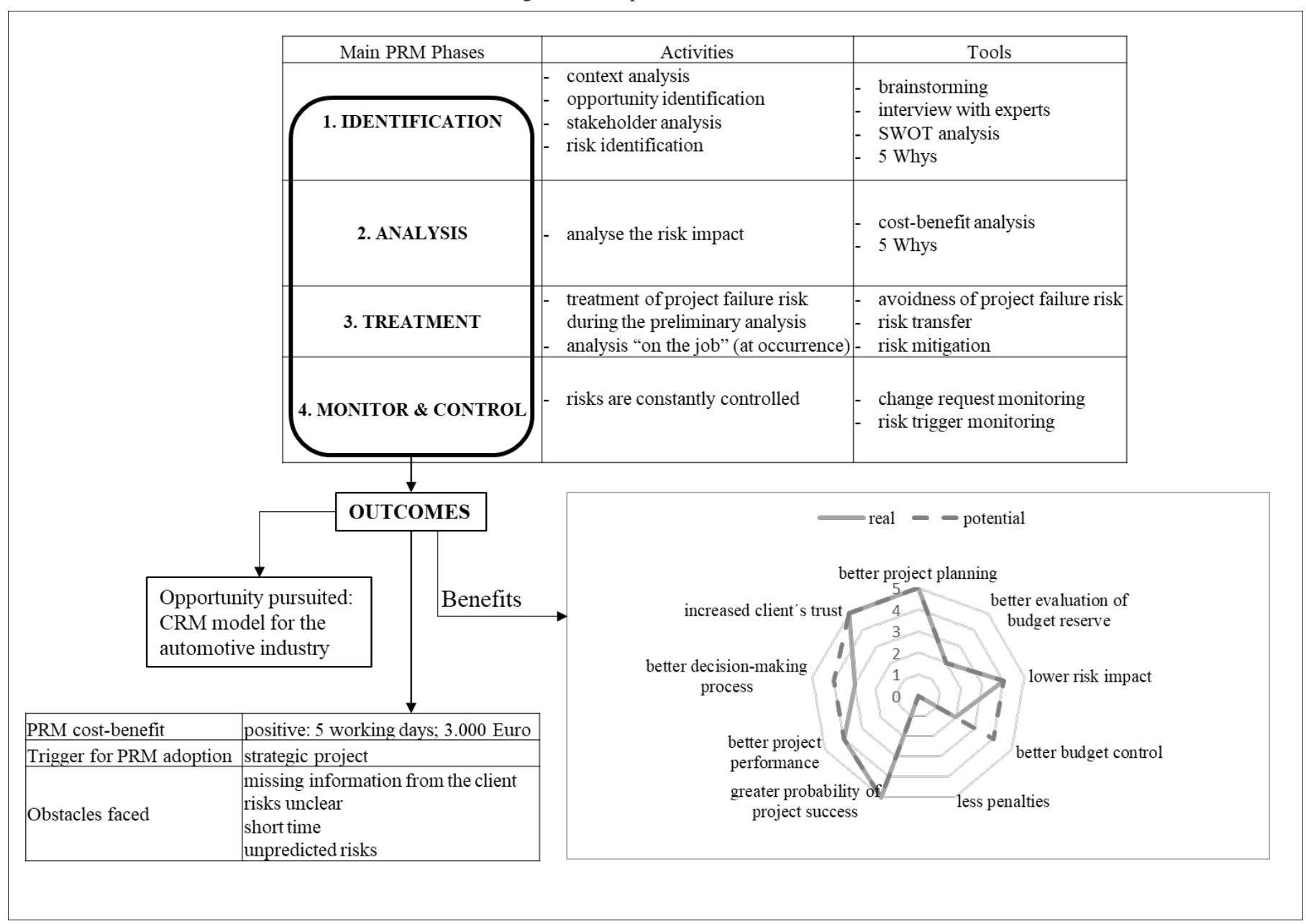




\section{Discussion}

The project analysed was successfully completed. From the analysis of the PRM, it was possible to grasp interesting evidence about how to adopt PRM in a SME. Firstly, besides all the qualitative benefits obtained from the PRM, it is important to highlight the positive costbenefit ratio between the RM outcomes and implementation costs, which can motivate the development of RM also in this type of company. Likewise, the study of Fernando et al. (2017) confirmed that PRM is positively related to PM performance.

In more details, Alpha devoted most of its efforts (i.e., in time and depth of the analysis) in identifying and dealing with the risks of project failure during the very early initiation phase of the project, in order to accept it only if project success is likely attended. This is realised in the preliminary analysis with a proactive approach, revealing the company knowledge and awareness that risks may lead to a complete project failure. Moreover, the project's success gave the company the important opportunity of captivating clients in a new market, which was identified and pursued because of the PRM. This result reinforced the affirmation of Carvalho and Rabechini (2015), who stated that PRM can use a mapping method of the threats and opportunities to develop new models.

This preliminary analysis also disclosed a positive characteristic of Alpha: a strategic vision. According to Carvalho and Rabechini (2015), PM needs to be embedded in strategic thinking on project risks to ensure the completion of the PM activities. The entire project team was involved in the PRM, which was also a positive result since, according to Radnor and Walley (2008), the entire staff should be involved and consulted in order to increase motivation and create conditions for a sustainable implementation.

The analysis also revealed that the main difficulties faced in Alpha's PRM process were the need of managing risks on the job, the unreliable information given by the client and the attempt to involve the client in the PRM process. Among the weak points, the following were highlighted: the lack of a risk register, the unstructured risk acceptance threshold definition (based on the consultants' feelings and experiences), the unwillingness of the top management to invest more time on PRM, the uncalculated cost of risk response and the absence of a document registering the lessons learned. Therefore, many decisions were purely based on the consultants' knowledge and experiences. When analysing the outcomes of the PRM adoption, the consultants presume that an improvement in the PRM process would significantly affect the benefits obtained.

In relation to the academic implications, the empirical framework developed for SMEs and tested in the pilot case allows researchers to analyse the PRM main dimensions and to determine the cost-benefit ratio connected with its adoption. After the pilot case, the questionnaire was improved: through its application it was possible to correct incomplete or unclear information and to complete the data collection requiring specific examples for every PM phase emerged. The resulting framework can give a valuable contribution to the literature since, to our best knowledge, this type of framework was not present. Regarding the practical implications, the Alpha case gives an example of an effective and efficient RM in the preliminary phase of project initiating, assuring project success; meanwhile, suggestions to improve the PRM have been highlighted, in order to improve project performance and fully benefit from the PRM adoption. Finally, this study confirmed that PRM adoption is SMEs is still at early stages, as emerged from the literature (Kim \& Vonortas, 2014).

\section{Conclusion}

Although this is a pilot case, limited to a specific industrial sector, it provided first indications about the PRM adoption in SMEs. The stra-tegic vision of Alpha is one of their main strengths. The company con-centrates its efforts in managing the risks of project failure to ensure the success of its projects, which is the main fundamental goal to be pursued through a PRM system, followed by optimising performance and extending a proactive approach to manage the other project risks that, if not carefully managed, often cause an increase in project costs and completion time.

As it emerged in the literature review, one of the reasons for SMEs to not apply PRM is that CEOs may believe its costs are not justifia-ble. However, the positive cost-benefit ratio obtained in this project shows that the benefits obtained from the PRM adoption overcome the related costs.

This pilot case verified the validity of and refined the proposed em-pirical framework. It could be used in future studies, which should focus on different types of industries, specifically considering the size of the company (i.e., small or medium) and the company's production organisation (i.e., whether the company is organised by process or by projects), besides the previously mentioned characteristics.

\section{Acknowledgements}

The authors gratefully acknowledge the support from the research projects of the University of Padova PANI_SID17_01

\section{References}

Badri, A. (2015). The Challenge of Integrating OHS into Industrial Project Risk Management: Proposal of a Methodological Approach to Guide Future Research (Case of Mining Projects in Quebec, Canada). Minerals, 5(2), 314-334.

Bajo, J., Borrajo, M. L., De Paz, J. F., Corchado, J. M., \& Pellicer, M. A. (2012). A multi-agent system for web-based risk management in small and medium business. Expert Systems with Applications, 39(8), 6921-6931.

Blanc Alquier, A. M., \& Lagasse Tignol, M. H. (2006). Risk manage-ment in small-and medium-sized enterprises. Production Planning \& Control, 17(3), 273-282.

Borge, D. (2002). The Book of Risk. New York, USA: John Wiley \& Sons. 
Cagliano, A. C., Grimaldi, S., \& Rafele, C. (2015). Choosing project risk management techniques. A theoretical framework. Journal of Risk Research, 18(2), 232-248.

Carvalho, M. M. D., \& Rabechini Junior, R. (2015). Impact of risk management on project performance: the importance of soft skills. International Journal of Production Research, 53(2), 321-340.

Dalcher, D. (2012). The nature of project management: a reflection on the anatomy of major projects by Morris and Hough. International Journal of Managing Projects in Business 5 (4), 643-660.

Dallago, B., Guglielmetti, C., \& Rondinelli, M. (2012). The consequences of the international crisis for European SMEs: vulnerability and resilience (vol. 27). Routledge.

Dey, P. K. (2012). Project risk management using multiple criteria decision-making technique and decision tree analysis: a case study of Indian oil refinery. Production Planning \& Control 23 (12), 903-921.

Elkington, P., \& Smallman, C. (2000). Managing Project Risks: a case study from the utilities sector. International Journal of Project Management, 20(1), 49-57.

Fernando, Y., Walters, T., Ismail, M. N., Seo, Y. W., \& Kaimasu, M. (2018). Managing project success using project risk and green supply chain management: A survey of automotive industry. International Journal of Managing Projects in Business, 11(2), 332-365.

Henschel, T. (2006). Risk management practices in German SMEs: an empirical investigation. Int. J. Entrepreneurship and Small Business, 3(5), 554-571.

ISO21500. (2012). Guidance on project management. International Organization for Standardization. http://www.iso.org.

ISO31000. (2009). International Organisation of Standardisation. Principles and generic guidelines on risk management. http://www. iso.org.

Kim, Y., \& Vonortas, N. S. (2014). Managing risk in the formative years: Evidence from young enterprises in Europe. Technovation, 34(8), 454-465.

Marcelino-Sádaba, S., Pérez-Ezcurdia, A., Lazcano, A. M., \& Villanueva, P. (2014). Project risk management methodology for small firms. International Journal of Project Management, 32(2), 327-340.

Miklosik, A. (2015). Improving project management performance through capability maturity measurement. Procedia Economics and Finance, 3, 522-530.
PMI. (2013). A Guide to Project Management Body of Knowledge: PMBoK Guide (Fifth Edition). Project Management Institute. USA.

PMI. (2017). A Guide to Project Management Body of Knowledge: PMBoK Guide (Sixth Edition). Project Management Institute. USA.

Radner, R., \& Shepp, L. (1996). Risk vs. profit potential: A model for corporate strategy. Journal of economic dynamics and Control, 20(8), 1373-1393.

Radnor, Z., \& Walley, P. (2008). Learning to walk before we try to run: adapting lean for the public sector. Public money and management, 28(1), 13-20.

Raz, T., \& Michael, E. (2001). Use and benefits of tools for project risk management. International journal of project management, 19(1), 9-17.

Shenhar, A. J., Milosevic, D., Dvir, D., \& Thamhain, H. (2007 (September)). Linking project management to business strategy. Project Management Institute.

Srivannaboon, S., \& Milosevic, D. Z. (2006). A two-way influence between business strategy and project management. International journal of project management, 24(6), 493-505.

Turner, J. R., Ledwith, A., \& Kelly, J. (2010). Project management in small to medium-sized enterprises: matching processes to the nature of the firm. International Journal of Project Management, 28(8), 744755.

Verbano, C., \& Venturini, K. (2011). Development paths of risk management: approaches, methods and fields of application. Journal of Risk Research, 14(5), 519-550.

Voss, C., Tsikriktsis, N., \& Frohlich, M. (2002). Case research in operations management. International journal of operations \& production management, 22(2), 195-219.

Yin, R. (1994). Case study research: design and methods. Newbury Park: SAGE Publications.

\section{Biographical notes}

Priscila Ferreira de Araujo Lima is $\mathrm{PhD}$ student in Engineering Management and Real Estate Economics at Department of Management and Engineering of the University of Padova. Her main research areas are Project Management and Risk Management.

Chiara Verbano is Associate Professor of Management and Engineering Economics at the Department of Management and Engineering of the University of Padova. Her major research interests are the fields of risk management and innovation management. 
